\title{
marges Marges
}

revue d'art contemporain Revue d'art contemporain

$02 \mid 2004$

Varia

\section{Deux expositions au Palais de Tokyo}

"Hardcore. Vers un nouvel activisme ", Site de création contemporaine du Palais de Tokyo, 27 février - 8 mai 2003 | « GNS. Global Navigation System », Site de création contemporaine du Palais de Tokyo, 5 juin 7 septembre 2003

Jérôme Glicenstein

\section{OpenEdition}

Journals

Édition électronique

URL : http://journals.openedition.org/marges/808

DOI : $10.4000 /$ marges. 808

ISSN : 2416-8742

Éditeur

Presses universitaires de Vincennes

Édition imprimée

Date de publication : 15 avril 2004

Pagination : 106-107

ISBN : 978-2-84292-246-7

ISSN : 1767-7114

\section{Référence électronique}

Jérôme Glicenstein, «Deux expositions au Palais de Tokyo », Marges [En ligne], 02 | 2004, mis en ligne le 06 août 2014, consulté le 22 septembre 2020. URL : http://journals.openedition.org/marges/808 ; DOI : https://doi.org/10.4000/marges.808

Ce document a été généré automatiquement le 22 septembre 2020.

(c) Presses universitaires de Vincennes 


\section{Deux expositions au Palais de Tokyo}

"Hardcore. Vers un nouvel activisme ", Site de création contemporaine du Palais de Tokyo, 27 février - 8 mai $2003 \mid$ « GNS. Global Navigation System ", Site de création contemporaine du Palais de Tokyo, 5 juin 7 septembre 2003

Jérôme Glicenstein

\section{RÉFÉRENCE}

" Hardcore. Vers un nouvel activisme », Site de création contemporaine du Palais de Tokyo, 27 février - 8 mai 2003

« GNS. Global Navigation System », Site de création contemporaine du Palais de Tokyo, 5 juin -7 septembre 2003

1 Les expositions « Hardcore » et " GNS », organisées respectivement par Jérôme Sans et Nicolas Bourriaud au Palais de Tokyo début 2003, fournissent une occasion de voir s'exprimer les positions respectives des deux co-directeurs du lieu quant à la pratique du commissariat d'exposition, tout en permettant de jeter un regard sur la première année d'existence de cette nouvelle institution parisienne.

2 En dépit de la différence des démarches adoptées par Sans et Bourriaud - et de la différence des thématiques et des propos qui les sous-tendent -, ces deux expositions recèlent de nombreux points communs. Les deux accrochages sont classiques et sobres ; les œuvres bien délimitées et isolées suggèrant une "distance contemplative" respectable avec le spectateur. Ces deux expositions semblent de ce point de vue entériner assez clairement la transformation progressive du « chantier» du Palais de Tokyo en un espace d'exposition assimilable de plus en plus à un white cube interchangeable, où les œuvres proposées "débordent» assez peu d'un espace institutionnel somme toute assez convenu.

3 Il y a bien sûr, objectera-t-on, telle installation de voitures calcinées, tels murs " défoncés " (les murs ayant été préalablement construits à cet effet), tel «filet» rappelant les toitures des prisons, voire la reconstitution d'une sorte d'habitat précaire 
ou l'installation d'une "maquette» proliférante. Mais tout cela reste assez sage. Au fond, il n'y aurait pas lieu de trouver là matière à polémique et on pourrait tout aussi bien se contenter de considérer qu'il s'agit là de deux expositions de plus, où l'on aura vu avec plaisir ou déplaisir telle ou telle œuvre. Si des questions se posent, ce n'est pas tant au vu des expositions elles-mêmes - expositions qui ne semblent pas particulièrement "remarquables "-, mais plutôt en raison des ambitions affichées dans les deux catalogues.

Dans l'introduction au catalogue d'« Hard-core ", Jérôme Sans insiste en effet sur l'idée d'un «nouvel activisme », sur le fait que les artistes présentés " infiltrent la réalité » et "qu'ils suscitent de vrais débats ». Selon l'auteur, "l'œuvre devient une matière à réagir, à interrogations, plus qu'à contemplation». Pourtant, simultanément, il est affirmé dans le même texte que l'art fonctionne comme «métaphore de positions de résistances » et que « l'action et l'œuvre des artistes tiennent lieu de manifeste [...] qui la plupart du temps reste dans la sphère symbolique de l'écriture artistique ». De manière assez troublante, Jérôme Sans qui a réalisé des entretiens avec les artistes pour le catalogue leur a systématiquement posé la question de la place de leur travail au sein $\mathrm{du}$ monde de l'art et d'une institution légitimante. Et le plus intéressant est sans doute que la plupart des artistes aient répondu en remarquant justement que l'exposition dans un lieu tel que le Palais de Tokyo contribuait à désamorcer leur propos en l'« esthétisant» (voir notamment les réponses de Jota Castro, AAA.Corp, Maurizio Cattelan, Alain Declercq, Gianni Motti, etc.).

5 Le même genre de décalage se retrouve avec l'article de Nicolas Bourriaud qui inaugure le catalogue de «GNS ». Ce dernier y décrit l'art contemporain comme une «zone offshore", comme une "pensée plastique» autonome, tout en affirmant simultanément qu'il s'agit désormais de "réaliser concrètement» des idées, de reconstruire en quelque sorte la réalité. « L'art contemporain, nous dit-il, se structure comme un banc de montage alternatif, qui réorganise les formes sociales ou culturelles et les insère dans d'autres types de scénarios ». Tout le propos de Bourriaud tourne ainsi autour d'une critique des modes de représentation "réalistes» ou " documentaires » en vigueur dans l'art contemporain international (ce qu'il nomme le " réalisme CNN ») ; au profit « d'une pensée plastique qui assume ses responsabilités » et d'un formalisme débarrassé de l'identification à Clement Greenberg. En quelque sorte, il s'agit de reprendre le projet de la modernité formaliste et notamment l'idée "que les formes plastiques sont capables de produire par elles-mêmes des significations, et qu'aucune d'entre elles n'est neutre ». Évidemment, ce plaidoyer pour une abstraction renouvelée, pour la forme et contre le signe, entre en contradiction avec tout un pan du même texte à propos des capacités des artistes à agir dans le social.

6 Mais cette contradiction qui est au cœur de "GNS ", et qui redouble celle de " Hardcore ", ne renvoie-t-elle pas d'abord à l'ambivalence de la position de ces deux " commissaires indépendants » amenés à diriger une des institutions publiques majeures de l'art contemporain en France ? On lira comme un symptôme de cette contradiction le texte de refus diffusé sur Internet par le groupe Syndicat potentiel, une association d'artistes invitée à «GNS » (qui figure d'ailleurs au catalogue), et qui dans une lettre ouverte rend compte des difficultés à faire accepter par le Palais de Tokyo des projets dans l'espace public.

7 Les deux expositions renvoient ainsi, semble-t-il, à la même difficulté et aux mêmes interrogations : comment énoncer des positions radicales, tout en acceptant de se plier 
à des formes conventionnelles forcément restrictives (le centre d'art, l'accrochage, l'exposition...) ; comment "agir dans le réel » dans un lieu "artificiel par nature ", comment échapper à la "représentation", tout en restant dans les limites d'un " théâtre social» aussi fortement connoté (historiquement, socialement, géographiquement...) que le Palais de Tokyo? 